\title{
Estado emocional y situaciones de estrés en árbitros de baloncesto
}

\author{
Emotional state and stress situations in basketball referees
}

\section{Estado emocional e situação de estresse em árbitros de basquete}

\author{
Jaenes, J. C.*; Bohórquez, M. R.**; Caracuel, J. C.**; López, A. M.** \\ * Universidad Pablo de Olavide. Centro Andaluz de Medicina del Deporte (CAMD) \\ ** Universidad de Sevilla.
}

\begin{abstract}
Resumen
El objetivo de este estudio fue crear un listado de situaciones de estrés pre y durante partido, basándonos en la experiencia personal de 59 árbitros de la liga LEB durante un Clinic de árbitros del grupo I de la Asociación Espańola de Árbitros de Baloncesto y la Federación Espańola de dicho deporte. El Listado de Situaciones Estresantes para el Arbitraje (LISEA) evalúa no sólo la intensidad de la experiencia emocional, sino también la direccionalidad de la misma- El instrumento mostró una alta fiabilidad general (.897). Los resultados ponen de manifiesto que dichas fuentes influyen de forma significativa y diferente según el momento competitivo, siendo más estresante durante el partido menos en un item. Igualmente se observan correlaciones entre la experiencia arbitral en el nivel I y diferentes situaciones evaluadas, siendo más numerosas las correlaciones negativas. La intervención psicológica debería tener en cuenta especialmente estos hallazgos sobre las fuentes de más impacto y en el momento competitivo en que tienen mayor influencia.

Palabras clave: estado emocional, estrés, árbitros de baloncesto. ABSTRACT

The objective of this study was to draw up a list of stress related situations both before and during the game, based on the experience of 59 LEB League referees who participated in a Clinic for Group 1 of the Spanish Association of Basketball Referees and the Spanish Basketball Federation. The List of Stress related situations for Referees (LISEA) not only assesses the emotional experience but also the directional consequences of stress. The survey showed a very high accuracy value (.897) Results show that different
\end{abstract}

stress sources have a varying influence depending on the moment in the competition, being most stressful during the game except for one item. At the same time, there is a correlation between the refereeing experience at level 1 and different situations assessed, with negative correlations being most common. Psychological intervention should bear in mind these findings in relation to the stress sources with highest impact and the competitive moment during which they exert most influence.

Key words: motional state, stress, Basketball referees RESUMO

O objetivo desse estudo foi de criar uma lista de situaçôes de estresse pré e durante um jogo, baseando na experiência pessoal de 59 árbitros da Liga LEB durante um Clinic de árbitros do grupo I da Associação Espanhola de Árbitros de Basquete e da Federação Espanhola do mesmo esporte. A lista de Situaçōes Estressantes para a arbitragem (LISEA) avalia nao só a intensidade da experiência emocional, mas também a direção da mesma. $\mathrm{O}$ instrumento mostrou uma alta fiabilidade geral (.897). Os resultados manifestam as referidas fontes de forma significativa e diferente de acordo com o momento competitivo sendo, mas estressante durante um jogo, menos em um item. Da mesma forma foi observada correlaçóes entre a experiência arbitral no nível I e diferentes situaçóes avaliadas, sendo mais numerosas as correlaçóes negativas. A intervenção psicológica deveria considerar especialmente o descoberto entre as fontes de impacto e no momento competitivo que tem maior influencia.

PALABRAS CHAVE: estado emocional, estress, árbitros de basquete.

\section{Introducción}

El estudio del arbitraje y el juicio deportivo ha sido uno de los campos de investigación menos explorados en la Psicología del Deporte a pesar de ser un colectivo de máxima importancia para el desarrollo de la actividad deportiva (Blasco, 1999; Cruz, 2001; González, 1999; Guillén, 2003; Guillén y Jiménez, 2001).

Diversos estudios sobre psicología aplicada al arbitraje proponen que algunas de las características deseables en árbitros o jueces deportivos serían un adecuado conocimiento del reglamento, el mantenimiento de una buena forma física

Correspondencia: José Carlos Jaenes. Departamento de Ciencias Sociales, Universidad Pablo de Olavide. Carretera de Utrera km 1. 41013, Sevilla. jcjaesan@upo.es y la colocación adecuada en el terreno de juego (Guillén y Jiménez, 2001); en el plano psicológico, se indican como características ideales ser consciente de las propias decisiones, establecer una buena relación con los otros, actuar con decisión y rapidez, mostrar un buen dominio y control emocional, actuar con integridad, tener autoconfianza y estar motivado y disfrutar durante la competición (Cruz, 2001; Weinberg y Richardson, 1990). Más recientemente Guillén y Feltz (2011) presentaron un modelo conceptual de eficacia y de las diferentes características que deben tener los árbitros para ser eficaces en su tarea, entendiendo esta como la creencia de tener la capacidad de arbitrar con éxito.

Porque los árbitros pueden sufrir enormes cantidades estrés (crónico y también agudo), los estudios sobre arbitraje en psicología del deporte han tratado ese aspecto de forma des- 
tacada (Alonso-Arbiol, 2005; González, 1999; Saldaña, Cuadras y Fernández, 1989), entendiendo que sería el factor que mayor influencia tendría sobre las características psicológicas deseables (Labrador, 1992). Este estrés específico en árbitros/ jueces derivaría de una situación vivenciada y evaluada como amenazante con base en el conocimiento que se tiene del reglamento así como de sus experiencias anteriores y habiendo realizado una evaluación subjetiva de acuerdo con un medio ambiente determinado (Samulski y Noce, 2003).

Las fuentes de estrés en árbitros/jueces más señaladas en la literatura científica son la comisión de errores, la aparición de situaciones de conflicto interpersonal con entrenadores y jugadores y las agresiones (eminentemente verbales) por parte de entrenadores, jugadores o público (Alonso-Arbiol, 2005). Dorsch y Paskevich (2007) indican que las experiencias de estrés en árbitros dependen de su nivel de arbitraje, siendo la relación con el compañero, la confrontación con los entrenadores y el miedo a cometer errores las fuentes más importantes de estrés. A estas clásicamente señaladas, cabría añadir las que se producen en los momentos inmediatamente anteriores al partido, tanto en el vestuario como en la salida al campo (Morilla, Caracuel y Benítez, 1997), e incluso otras posteriores al mismo (Cruz, 1997; González, 1999). Como señalan Goldsmith y Williams (1992), sería necesario analizar las diferentes fuentes de estrés y los diferentes momentos temporales en que éstas aparecen, teniendo en cuenta que serán cambiantes en función de la modalidad deportiva y el nivel de competición.

Conocer las situaciones estresantes y las manifestaciones de estrés asociadas a éstas puede constituir un punto de partida sobre el que articular intervenciones dirigidas a la mejora de las habilidades psicológicas relacionadas con el arbitraje, permitiendo la identificación de situaciones adecuadas para la intervención (Blasco, 1999; González, 1999). Así, el objetivo principal de este estudio fue el diseño de una escala de medida de las situaciones estresantes propias del arbitraje de baloncesto que reflejara tanto la intensidad de la incidencia de éstas como la dirección de dicha intensidad. Además, se pretendía que la escala fuera sensible a las situaciones precompetitiva y competitiva.

\section{Método}

\section{Participantes}

La muestra estuvo compuesta por 59 árbitros de la Liga LEB, 57 hombres y 2 mujeres, que componían el total de la población de árbitros de esta liga. La edad media fue 32.22 años (DT = 4.483). Llevaban arbitrando 16.237 años de media (DT = 4.996), de los cuales habían desempeñado esta labor en el Nivel I 4.559 años de media (DT = 3.688).

\section{Instrumentos}

Se administró a los participantes un Listado de Situaciones Estresantes para el Arbitraje compuesto por 15 ítemes, seleccionados de entre los listados que ellos mismos habían confeccionado como parte de un ejercicio en el clinic y que los participantes debían puntuar en sus dimensiones intensidad y direccionalidad.

Este Listado de Situaciones Estresantes fue diseñado ad hoc partiendo de afirmaciones propias de los árbitros al realizar una auto-observación de sus estados emocionales antes de y durante los encuentros; durante su elaboración se siguió el procedimiento recomendado por Anshel y Weinberg (1995).

Las instrucciones dadas a los participantes fueron: "A continuación encontrarás una serie de situaciones (personas, eventos, etc.) que pueden influir en el estado emocional del árbitro. Nos gustaria saber en qué medida (intensidad) le afectan a usted cada una de ellas, siendo 0 no me afecta en absoluto y 10 me afecta en gran medida. Al mismo tiempo, nos gustaría saber si estas situaciones le ayudan o le perjudican (direccionalidad) en su rendimiento en la cancha; en este caso 0 me afecta negativamente y 10 me sirve para hacerlo mucho mejor, siendo 5 un punto medio." Además, en las instrucciones se indicaba el momento temporal (antes o durante el partido) en el que debían evaluar la incidencia de dichas situaciones estresantes.

La tabla 1 muestra el instrumento en su formato final:

Tabla 1. Listado de Situaciones Estresantes para el Arbitraje (LISEA).

\begin{tabular}{|c|c|c|c|}
\hline & \multicolumn{2}{|c|}{ GRADO EN QUE ME AFECTA. } & SENTIDO EN QUE ME AFECTA \\
\hline & $\begin{array}{c}0 \\
\text { "nada" }\end{array}$ & $\begin{array}{c}10 \\
\text { "en gran medida" }\end{array}$ & $\begin{array}{cc}0 & 10 \\
\text { "muy negativamente" "muy positivamente" }\end{array}$ \\
\hline \multicolumn{4}{|l|}{ Nombre del entrenador. } \\
\hline \multicolumn{4}{|l|}{ Nombre del equipo. } \\
\hline \multicolumn{4}{|l|}{ Nombre del jugador. } \\
\hline \multicolumn{4}{|l|}{ Puesto que ocupa en la competición. } \\
\hline \multicolumn{4}{|l|}{ Presencia de un informador. } \\
\hline \multicolumn{4}{|l|}{ Presencia de los medios de comunicación. } \\
\hline Volumen de público. & & & \\
\hline
\end{tabular}




\begin{tabular}{|c|c|c|c|}
\hline & \multicolumn{2}{|c|}{ GRADO EN QUE ME AFECTA. } & SENTIDO EN QUE ME AFECTA \\
\hline & $\begin{array}{c}0 \\
\text { "nada” }\end{array}$ & $\begin{array}{c}10 \\
\text { "en gran medida" }\end{array}$ & $\begin{array}{cc}0 & 10 \\
\text { "muy negativamente" "muy positivamente" }\end{array}$ \\
\hline \multicolumn{4}{|l|}{ Ruido en el pabellón. } \\
\hline \multicolumn{4}{|l|}{ Compañero de arbitraje. } \\
\hline \multicolumn{4}{|l|}{ Localidad lejos de casa. } \\
\hline \multicolumn{4}{|l|}{ Tipo de desplazamiento. } \\
\hline \multicolumn{4}{|l|}{ Pitar en campo desconocido. } \\
\hline \multicolumn{4}{|l|}{ Persona que el club pone a tu disposición. } \\
\hline \multicolumn{4}{|l|}{ Situación pre-partido. } \\
\hline Actuaciones arbitrales previas & & & \\
\hline
\end{tabular}

\section{Procedimiento}

La investigación se llevó a cabo durante un Clínic de Árbitros de Baloncesto del Grupo I organizado por la Federación Española de Baloncesto, la Asociación Española de Árbitros de Baloncesto y la colaboración del Centro Andaluz de Medicina del Deporte (CAMD). Este Clínic, que servía de preparación para la temporada, acogía un taller sobre Aspectos Psicológicos del Arbitraje que tenía como objetivo mejorar el rendimiento en competición de los participantes.

Durante la realización de este clínic se pidió a los árbitros que cumplimentaran de forma anónima el Listado de Situaciones Estresantes para el Arbitraje, valorando las dimensiones intensidad y direccionalidad del estado emocional consecuente en los dos momentos temporales señalados. No se explicitó un tiempo límite para la realización, aunque se les invitó a aportar la primera valoración que se les ocurriera tratando de ser lo más sinceros posible.

Una vez cumplimentado el listado se procedió al análisis del mismo por parte de los participantes, estando asistidos por el psicólogo a cargo del Clínic. Finalmente, se pidió a los árbitros que, voluntariamente, cedieran sus Listados completados con fines de investigación.

\section{Resultados}

\section{Análisis descriptivos.}

En la Tabla 2 se muestran los estadísticos descriptivos (mínimos, máximos, media y desviación típica) de los ítems de la escala de situaciones de estrés en árbitros de baloncesto.

Tabla 2. Estadísticos descriptivos de los ítems de la escala de estrés en árbitros de baloncesto.

\begin{tabular}{|c|c|c|c|c|c|}
\hline & Ítem & Mínimo & Máximo & Media & DT \\
\hline \multirow{15}{*}{ 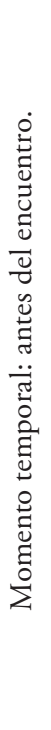 } & Nombre del entrenador. & .00 & 9.00 & 1.97 & 2.04 \\
\hline & Nombre del equipo. & .00 & 8.00 & 2.07 & 2.20 \\
\hline & Nombre del jugador. & .00 & 7.00 & 1.54 & 1.91 \\
\hline & Puesto que ocupa en la liga. & .00 & 8.00 & 1.97 & 2.28 \\
\hline & Presencia de un informador. & .00 & 8.00 & 2.84 & 2.58 \\
\hline & Presencia de los medios de comunicación. & .00 & 9.00 & 1.64 & 2.24 \\
\hline & Volumen de público. & .00 & 10.00 & 3.11 & 2.92 \\
\hline & Ruido en el pabellón. & .00 & 10.00 & 2.92 & 2.95 \\
\hline & Compañero de arbitraje. & .00 & 10.00 & 3.40 & 2.74 \\
\hline & Localidad lejos/cerca de casa. & .00 & 10.00 & 1.49 & 2.28 \\
\hline & Tipo de desplazamiento. & .00 & 10.00 & 2.20 & 2.47 \\
\hline & Pitar en campo desconocido. & .00 & 8.00 & 1.61 & 1.96 \\
\hline & Persona que el club pone a tu disposición. & .00 & 6.00 & .61 & 1.35 \\
\hline & Situación pre-partido. & .00 & 8.00 & 2.68 & 2.39 \\
\hline & Actuaciones arbitrales previas. & .00 & 9.00 & 3.49 & 2.53 \\
\hline
\end{tabular}




\begin{tabular}{|c|c|c|c|c|c|}
\hline & Ítem & Mínimo & Máximo & Media & DT \\
\hline \multirow{14}{*}{ 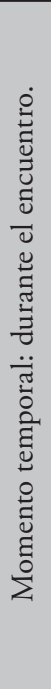 } & Nombre del entrenador. & .00 & 10.00 & 5.91 & 1.68 \\
\hline & Nombre del equipo & .00 & 10.00 & 6.07 & 1.90 \\
\hline & Nombre del jugador. & .00 & 10.00 & 5.56 & 2.04 \\
\hline & Puesto que ocupa en la liga. & .00 & 10.00 & 5.93 & 2.06 \\
\hline & Presencia de un informador. & .00 & 10.00 & 6.15 & 1.95 \\
\hline & Presencia de los medios de comunicación. & .00 & 10.00 & 6.24 & 1.99 \\
\hline & Volumen de público. & 2.00 & 10.00 & 7.80 & 1.84 \\
\hline & Ruido en el pabellón. & .00 & 10.00 & 7.44 & 2.23 \\
\hline & Compañero de arbitraje. & .00 & 10.00 & 5.86 & 1.90 \\
\hline & Localidad lejos/cerca de casa. & .00 & 10.00 & 5.20 & 1.79 \\
\hline & Tipo de desplazamiento. & .00 & 8.00 & 4.81 & 1.50 \\
\hline & Pitar en campo desconocido. & .00 & 10.00 & 4.97 & 1.74 \\
\hline & Persona que el club pone a tu disposición. & .00 & 8.00 & 4.83 & 1.10 \\
\hline & Situación pre-partido. & .00 & 10.00 & 5.58 & 1.84 \\
\hline
\end{tabular}

\section{Análisis de frabilidad}

La fiabilidad general de la escala de situaciones estresantes para árbitros de baloncesto fue medida mediante el alfa de Cronbach, obteniendo una puntuación de .897 , mostrando por lo tanto una alta consistencia interna.

Además del cálculo de la consistencia interna general de la escala, se llevó a cabo un análisis de la fiabilidad de la misma en caso de eliminar algún ítem. La Tabla 3 muestra que la fiabilidad de la escala sólo se vería beneficiada de la eliminación del ítem 10 "arbitrar en una localidad lejos/cerca de casa", aunque no lo haría de manera ostensible, por lo que se decidió no suprimirlo de la escala.

Tabla 3. Fiabilidad de la escala si se elimina algún ítem.

Ítem

Alfa de Cronbach si se elimina el elemento.

\begin{tabular}{ll}
\hline Nombre del entrenador. & .886 \\
Nombre del equipo. & .885 \\
Nombre del jugador. & .886 \\
\hline Puesto que ocupa en la liga. & .889 \\
\hline Presencia de un informador. & .892 \\
\hline Presencia de los medios de comunicación. & .888 \\
\hline Volumen de público. & .886 \\
Ruido en el pabellón. & .886 \\
Compańero de arbitraje. & .892 \\
\hline Localidad lejos/cerca de casa. & .900 \\
Tipo de desplazamiento. & .895
\end{tabular}

Ítem

Alfa de Cronbach

si se elimina el elemento.

\begin{tabular}{ll}
\hline Pitar en campo desconocido. & .890 \\
Persona que el club pone a tu disposición. & .895 \\
Situación pre-partido. & .889 \\
Actuaciones arbitrales previas. & .892 \\
\hline
\end{tabular}

Análisis de correlaciones.

Se analizó la relación entre los ítems de la escala de situaciones de estrés y los años de antigüedad arbitrando en el Nivel I utilizando el coeficiente de correlación de Pearson, al haberse hipotetizado que habría correlación entre estas variables.

Los resultados (ver tabla 4) muestran correlaciones significativas entre los años arbitrando en el Nivel I y los ítems presencia de un informador (correlación negativa), cercaniallejanía de la localidad donde se arbitra (correlación positiva, a mayor distancia de la localidad donde se arbitra mayor incidencia de estrés) y actuaciones arbitrales previas (correlación negativa).

Tabla 4. Análisis de correlaciones escala-años en el Nivel I.

\begin{tabular}{lc}
\hline Ítem & Correlación Pearson. \\
\hline Nombre del entrenador. & -.138 \\
Nombre del equipo. & -.161 \\
Nombre del jugador. & -.114 \\
Puesto que ocupa en la liga. & -.158 \\
Presencia de un informador. & $-.309^{*}$ \\
Presencia de los medios de comunicación. & -.050 \\
Volumen de público. & -.231
\end{tabular}




\begin{tabular}{lc}
\hline Ítem & Correlación Pearson. \\
\hline Ruido en el pabellón. & -.176 \\
Compañero de arbitraje. & -.055 \\
Localidad lejos/cerca de casa. & $.339^{* *}$ \\
\hline Tipo de desplazamiento. & .023 \\
\hline Pitar en campo desconocido. & -.178 \\
\hline Persona que el club pone a tu disposición. & .099 \\
Situación pre-partido. & -.140 \\
\hline Actuaciones arbitrales previas. & $-.256^{*}$ \\
\hline${ }^{*}$ La correlación es significativa a nivel 0.05 (bilateral). \\
${ }^{* *}$ La correlación es significativa a nivel 0.01 (bilateral).
\end{tabular}

También es posible apreciar que, aunque la mayor parte de las correlaciones no son significativas, sí existe una mayor cantidad de relaciones negativas.

Con el fin de determinar si las distintas fuentes de estrés influían de forma diferente en los distintos momentos temporales propuestos (antes y durante el encuentro), se realizó un análisis de correlación utilizando la prueba t de Student. Como muestra la Tabla 5, se encontraron correlaciones significativas en todos los ítems (pares antes-durante).

Tabla 5. Correlación por pares de cada ítem antes-durante el encuentro.

\begin{tabular}{lccc}
\hline Ítem & $\mathrm{t}$ & $\mathrm{gl}$ & Sig. (bilateral) \\
\hline Nombre del entrenador. & -13.73 & 58 & $.00^{* *}$ \\
\hline Nombre del equipo. & -14.68 & 58 & $.00^{* *}$ \\
Nombre del jugador. & -12.47 & 58 & $.00^{* *}$ \\
\hline Puesto que ocupa en la liga. & -12.05 & 58 & $.00^{* *}$ \\
Presencia de un informador. & -9.47 & 58 & $.00^{* *}$ \\
Presencia de los medios de comunicación. & -12.95 & 58 & $.00^{* *}$ \\
Volumen de público. & -11.05 & 58 & $.00^{* *}$ \\
Ruido en el pabellón. & -10.04 & 58 & $.00^{* *}$ \\
Compańero de arbitraje. & -6.60 & 58 & $.00^{* *}$ \\
\hline Localidad lejos/cerca de casa. & -11.16 & 58 & $.00^{* *}$ \\
Tipo de desplazamiento. & -6.81 & 58 & $.00^{* *}$ \\
Pitar en campo desconocido. & -20.42 & 58 & $.00^{* *}$ \\
Persona que el club pone a tu disposición. & -8.61 & 58 & $.00^{* *}$ \\
Situación pre-partido. & -9.77 & 58 & $.00^{* *}$ \\
Actuaciones arbitrales previas. & -6.24 & 58 & $.00^{* *}$ \\
\hline
\end{tabular}

**La correlación es significativa a nivel 0.01 (bilateral).

La incidencia de las diferentes situaciones estresantes sobre los árbitros antes de los encuentros resultó ser significativamente más baja que dicha incidencia durante los encuentros salvo para el ítem 13 persona que el club pone a tu disposición, cuya incidencia fue mayor en los momentos previos al partido que durante éste (Figura 1). 
Figura 1. Incidencia de situaciones estresantes antes y durante el partido.

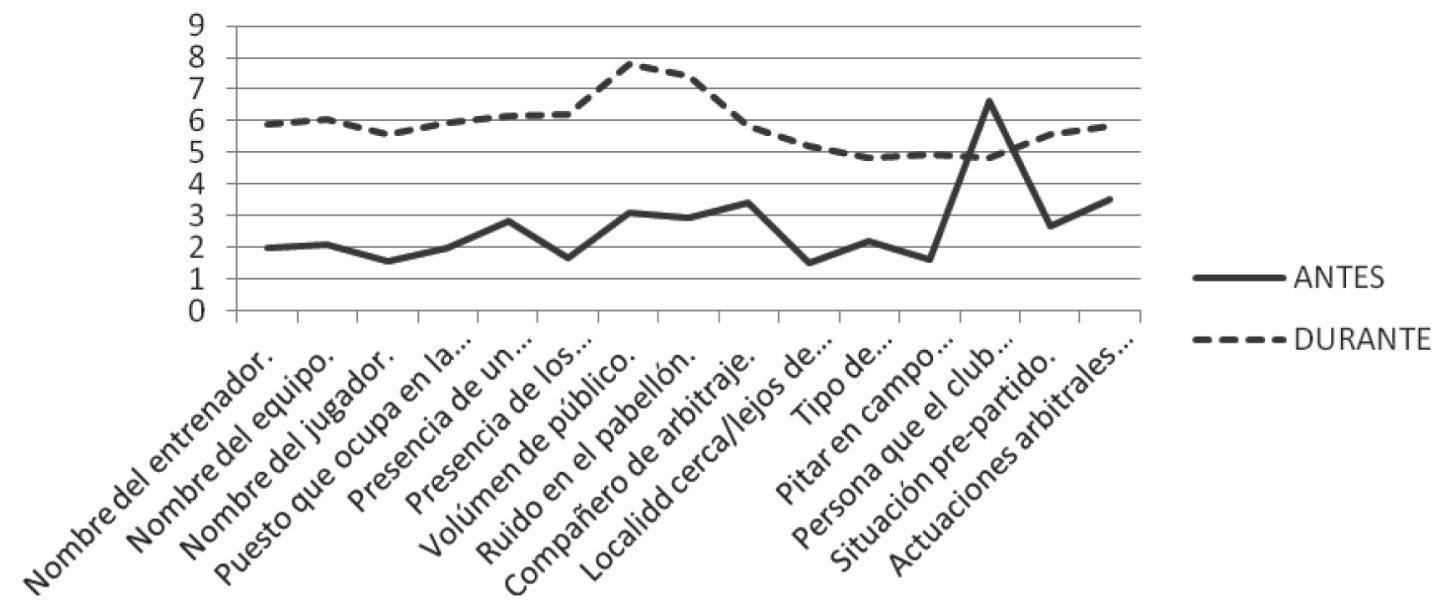

\section{Discusión}

El Listado de Situaciones Estresantes para el Arbitraje (LISEA) ha mostrado una alta fiabilidad (.897), considerándose por su consistencia interna una escala unidimensional. Esta alta fiabilidad puede explicarse en base a su construcción, guiada por el discurso que los propios árbitros mantenían durante el clinic acerca de esas situaciones que les resultaban estresantes.

Además, la escala ha mostrado ser sensible al impacto que las diferentes fuentes de estrés pudieran tener en diferentes momentos temporales (antes y después del encuentro a arbitrar), siendo esta medición en diferentes momentos la principal diferencia entre este instrumento y otros como el Cuestionario sobre Arbitraje Deportivo de González (1999), el Cuestionario de Estrés en Árbitros de Fútbol de AlonsoArbiol (2005) o el Cuestionario para Árbitros de Fútbol de Buceta, del Pino, Gimeno, García-Aranda y Ruíz (1999).

Concretamente, se han encontrado puntuaciones moderadas de estrés en los momentos previos al partido y bajas puntuaciones de estrés durante éste, como ya encontraron en sus estudios Blasco (1999), Goldsmith y Williams (1992) o González (1999). Este último dato ha sido tradicionalmente explicado con base en la relación negativa que los niveles de estrés soportado mantenían con la experiencia en el arbitraje, lo que permitiría a los colegiados manejar eficazmente sus recursos de afrontamiento (Dorsch y Paskevich, 2007; Guillén y Jiménez, 2001; Kassidis y Anshel, 1993; Rainey, 1995). Esta correlación negativa es significativa especialmente en lo que se refiere a la presencia de un informador y las actuaciones arbitrales previas; probablemente la historia interactiva sea el principal factor modulador de esta correlación.

De lo anterior se deduce la necesidad de formación en aspectos psicológicos que inciden en el comportamiento del árbitro (Caracuel, 2003; Caracuel, Andréu y Pérez, 1995), especialmente para los árbitros noveles. Entre los contenidos destacados para formar parte de una intervención formativa con árbitros se proponen como prioritarias las destrezas para el control emocional, estrategias de afrontamiento eficaces, control de ansiedad y estrés (Ramírez, Alonso-Arbiol, Falcó y López, 2006). Sería interesante que este tipo de intervenciones formativas se llevara a cabo en los colegios de árbitros tal y como sugieren Cantón, Gistain, Checa y León (2011) o Guillén y Jiménez (2001).

Investigaciones futuras deberían abordar listados de situaciones estresantes para las diferentes modalidades deportivas, puesto que el carácter situacional de los estresores puede derivar en cambios en los ítems señalados en el LISEA según deportes.

\section{Referencias}

1. Alonso-Arbiol, I.; Falcó, F.; López, M.; Ordaz, B. y Ramírez, A. (2005). Development of a questionnaire for the assesment of sources of stress in spanish soccer referees. Ansiedad y Estrés, 11(2-3), 175-188.

2. Anshel, M. H. y Weinberg, R. S. (1995). Sources of stress in American and Australian basketball referees. Journal of Applied sport Psychology, 7, 11-22.
3. Blasco, T. (1999). Competencia personal, autoeficacia y estrés en árbitros de ciclismo. Revista de Psicología del Deporte, 8(2), 195-205.

4. Buceta, J. M.; del Pino, C.; Gimeno, F.; García-Aranda, J. M.; Ruíz, J. A. (1999). Necesidades psicológicas de los árbitros de fútbol de alta competición. Tomado de http://www.palestraweb.com/zona_libre/boletin/ carpenews05/arbit.htm 
5. Cantón, E.; Gistain, C.; Checa, I.; León, E. (2011). Programa de mediación psicológica con árbitros de fútbol. SOCIOTAM, aceptado en proceso de edición.

6. Caracuel, J. C. (2003). Funciones psicológicas implicada en el arbitraje y el juicio deportivo. En F. Guillén (Ed.), Psicología del arbitraje y el juicio deportivo (pp.25-37). Barcelona: INDE.

7. Caracuel, J. C.; Andreu, R. y Pérez, E. (1995). Análisis psicológico del arbitraje y el juicio deportivos: una aproximación desde el modelo interconductual. Motricidad, 1, 5-24.

8. Cruz, J. (2001). Asesoramiento psicológico en el arbitraje y el juicio deportivos. En J. Cruz (Ed.), Psicología del Deporte (pp. 245-269). Madrid: Síntesis.

9. Dorsch, K. y Paskevich, D. (2007). Stressful experiences among six certification levels of ice hockey officials. Psychology of Sport and Exercise, 8, 585-593.

10. Goldsmith, P. A. y Wiliams, J. M. (1992). Perceived stressors for football and volleyball officials from three rating levels. Journal of Sport Behavior, 15(2), 106-118.

11. González, A. M. (1999). Magnitud y fuentes de estrés en árbitros de baloncesto y voleibol de élite. En G. Nieto y E. Garcés (coord.). Psicología de la actividad física y el deporte: Áreas de investigación y aplicación (pp. 52-59). Murcia: Sociedad Murciana de Psicología de la Actividad Física y el Deporte.

12. Guillén, F. Feltz, DL. (2011). A conceptual modelo of referee efficacy. Front. Psychology, 2:25. doi: 10.3389/fpsyg.2011.00025.
13. Guillén, F. y Jiménez, H. (2001). Características deseables en el arbitraje y el juicio deportivo. Revista de Psicología del Deporte, 10 (1), 23-24.

14. Guillén, F. (2003). Panorama actual en el estudio del arbitraje y el juicio deportivo desde una perspectiva psicológica. En F. Guillén (Ed.), Psicología del arbitraje y el juicio deportivo (pp.7-24). Barcelona: INDE.

15. Kassidis, A. y Anshel, M. H. (1993). Sources of and responses to acute stress in adult and adolescent Australian basketball referees. Australian Journal of Science and Medicine in Sport, 25, 97-103.

16. Labrador, F. J. (1992). El estrés. Madrid: Temas de hoy.

17. Rainey, D. W. (1995). Stress, burnout and intention to terminate among umpires. Journal of Sport Behavior, 18(4), 312-323.

18. Ramírez, A.; Alonso-Arbiol, I., Falcó, F.; López, M. (2006). Programa de intervención psicológica con árbitros de fútbol. Revista de Psicología del Deporte 15(2), 311-325.

19. Saldaña, C.; Cuadras, A.; Fernández, J. (1989). Entrenamiento en inoculación de estrés en árbitros de baloncesto: un estudio piloto. Actas de las V Jornadas de l'Assocciació Catalana de Psicología de l'Esport. Barcelona, 25-26 de Noviembre de 1998, 111-116.

20. Samulski, D. M. y Noce, F. (2003). Estrés psicológico en árbitros de deportes colectivos. En F. Guillén (Ed.), Psicología del arbitraje y el juicio deportivo (pp.109-132). Barcelona: INDE.

21. Weinberg, R. S.; Richardson, P.A. (1990). Psychology of Officiating. Champaign, IL.: Leisure Press. 